DAPNIA / SPhN 96-20

TTP96-29

\title{
PROSPECTS FOR MEASURING $\Delta g$ FROM JETS AT HERA WITH POLARIZED PROTONS
}

\author{
J. FELTESSE, F. KUNNE \\ DAPNIA, CE Saclay, F91191 Gif/Yvette, France \\ E. MIRKES \\ Inst. f. Theor. Teilchenphysik, Universität Karlsruhe, D-76128 Karlsruhe, \\ Germany
}

\begin{abstract}
The measurement of the polarized gluon distribution function $\Delta g(x)$ from photon gluon fusion processes in electron proton deep inelastic scattering producing two jets has been investigated. The study is based on the MEPJET simulation program. The size of the expected spin asymmetry and corresponding statistical uncertainties for a possible measurement with polarized beams of electrons and protons at HERA have been estimated. The results show that the asymmetry can reach a few percents.
\end{abstract}

\section{Introduction}

After confirmation of the surprising EMC result, that quarks carry only a little fraction of the nucleon spin, this subject is actively being studied by several fixed target experiments at CERN, DESY and SLAC 1 Sofar only the polarized structure functions $g_{1}\left(x_{g}, Q^{2}\right)$ and $g_{2}\left(x_{g}, Q^{2}\right)$ have been measured. These do not allow to distinguish between the role of quarks and gluons distributions. The measurement of the polarized gluon distribution $\Delta g\left(x_{g}, Q^{2}\right)$ has become the key experiment in order to understand the QCD properties of the spin of the nucleon. We study here the possible direct measurement of $\Delta g\left(x_{g}, Q^{2}\right)$ from jet events at the HERA collider, in the scenario where both the electron and the proton beam are polarized.

The gluon distribution enters at leading order (LO) in the two-jets production cross section 7 in deep inelastic scattering (DIS) (see Fig.1), and the unpolarized gluon distribution $g\left(x_{g}, Q^{2}\right)$ has indeed already been extracted from two-jets events by the H1 collaboration at HERA. With the modest luminosity of $0.24 p^{-1}$ obtained in 1993, first data on $x_{g} g\left(x_{g}\right)$ were extracted from dijets events $\mathrm{O}$ at $\mathrm{LO}$, in a wide $x_{g}$ range $0.002<x_{g}<0.2$, at a mean $Q^{2}$ of $30 \mathrm{GeV}^{2}$. These results happen to be in good agreement with the gluon distribution extracted at $\mathrm{LO}$ from scaling violations of the structure function $F_{2}$.

\footnotetext{
${ }^{a}$ In the following the jet due to the beam remnant is not included in the number of jets.
} 

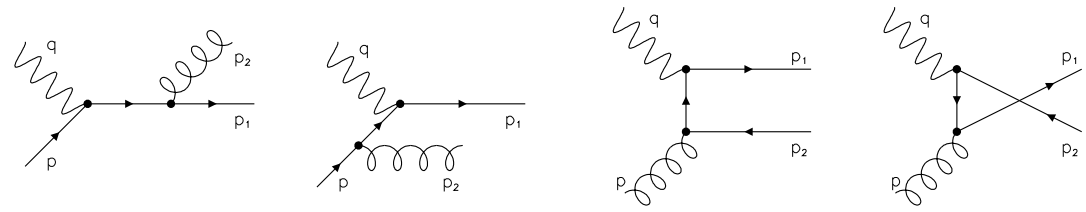

Figure 1: Feynman diagrams for the dijet cross section at LO.

\section{Jet cross sections in DIS}

Deep inelastic electron proton scattering with several partons in the final state,

$$
e^{-}(l)+p(P) \rightarrow e^{-}\left(l^{\prime}\right)+\operatorname{remnant}\left(p_{r}\right)+\text { parton } 1\left(p_{1}\right)+\ldots+\text { parton } n\left(p_{n}\right)
$$

proceeds via the exchange of an intermediate vector boson $V=\gamma, Z$. In the following, $Z$-exchange will be neglected. We denote the momentum of the virtual photon, $\gamma^{*}$, by $q=l-l^{\prime}$, its absolute square by $Q^{2}$, and use the standard scaling variables $x_{B j}=Q^{2} /(2 P \cdot q)$ and $y=P \cdot q / P \cdot l$. The general structure of the unpolarized $n$-jet cross section in DIS is given by

$$
d \sigma^{h a d}[n-\text { jet }]=\sum_{a} \int d x_{a} f_{a}\left(x_{a}, \mu_{F}^{2}\right) d \hat{\sigma}^{a}\left(p=x_{a} P, \alpha_{s}\left(\mu_{R}^{2}\right), \mu_{R}^{2}, \mu_{F}^{2}\right)
$$

where the sum runs over incident partons $a=q, \bar{q}, g$ which carry a fraction $x_{a}$ of the proton momentum. $\hat{\sigma}^{a}$ denotes the partonic cross section from which collinear initial state singularities have been factorized out (in next-toleading order (NLO)) at a scale $\mu_{F}$ and implicitly included in the scale dependent parton densities $f_{a}\left(x_{a}, \mu_{F}^{2}\right)$. For polarized lepton hadron scattering, the hadronic $(n$-jet) cross section is obtained from Eq. (2) with the replacements $\left(\sigma^{h a d}, f_{a}, \hat{\sigma}^{a}\right) \rightarrow\left(\Delta \sigma^{h a d}, \Delta f_{a}, \Delta \hat{\sigma}^{a}\right)$. The polarized hadronic cross section is 
defined by $\Delta \sigma^{\text {had }} \equiv \sigma_{\uparrow \uparrow}^{\text {had }}-\sigma_{\uparrow \downarrow}^{\text {had }}$, where the left arrow in the subscript denotes the polarization of the incoming lepton with respect to the direction of its momentum. The right arrow stands for the polarization of the proton parallel or anti-parallel to the polarization of the incoming lepton. The polarized parton distributions are defined by $\Delta f_{a}\left(x_{a}, \mu_{F}^{2}\right) \equiv f_{a \uparrow}\left(x_{a}, \mu_{F}^{2}\right)-f_{a \downarrow}\left(x_{a}, \mu_{F}^{2}\right)$. Here, $f_{a \uparrow}\left(f_{a \downarrow}\right)$ denotes a parton $a$ in the proton whose spin is aligned (anti-aligned) to the proton's spin. $\Delta \hat{\sigma}^{a}$ is the corresponding polarized partonic cross section.

In Born approximation, the subprocesses $\gamma^{*}+q \rightarrow q+g, \gamma^{*}+\bar{q} \rightarrow \bar{q}+g$, $\gamma^{*}+g \rightarrow q+\bar{q}$ contribute to the two-jet cross section (Fig.(1). The boson gluon fusion subprocess $\gamma^{*}+g \rightarrow q+\bar{q}$ dominates the two jet cross section at low $x_{B j}$ (see below) and allows for a direct measurement of the gluon density in the proton. The full NLO corrections for two jet production in unpolarized lepton hadron scattering are now available 3 and implemented in the $e p \rightarrow n$ jets event generator MEPJET, which allows to analyze arbitrary jet definition schemes and general cuts in terms of parton 4-momenta.

First discussions about jet production in polarized lepton-hadron scattering can be found in Ref. [4], where the jets were defined in a modified "JADE" scheme. However, it was found 3 that the theoretical uncertainties of the twojet cross section for the "JADE" scheme can be very large due to higher order effects. These uncertainties are small for the cone scheme and the following results are therefore based on the cone algorithm, which is defined in the laboratory frame. In this algorithm the distance $\Delta R=\sqrt{(\Delta \eta)^{2}+(\Delta \phi)^{2}}$ between two partons decides whether they should be recombined to a single jet. Here the variables are the pseudo-rapidity $\eta$ and the azimuthal angle $\phi$. We recombine partons with $\Delta R<1$. Furthermore, a cut on the jet transverse momenta of $p_{T}(j)>5 \mathrm{GeV}$ in the lab frame and in the Breit frame is imposed. We employ the one loop (two loop) formula for the strong coupling constant in a LO (NLO) analyses with a value for $\Lambda \frac{(4)}{M S}$ consistent with the value from the parton distribution functions. In addition a minimal set of general kinematical cuts is imposed on the virtual photon and on the final state electron and jets. If not stated otherwise, we require $5 \mathrm{GeV}^{2}<Q^{2}<2500 \mathrm{GeV}^{2}, 0.3<y<1$, an energy cut of $E\left(e^{\prime}\right)>5 \mathrm{GeV}$ on the scattered electron, and a cut on the pseudo-rapidity $\eta=-\ln \tan (\theta / 2)$ of the scattered lepton (jets) of $|\eta|<3.5$ $(|\eta|<2.8)$. These cuts are compatible with the existing detectors H1 and ZEUS.

Let us briefly discuss the choice of the renormalization and factorization scales $\mu_{R}$ and $\mu_{F}$ in Eq. (2). Jet production is a multi-scale problem and it is not a priori clear at which scale $\alpha_{s}$ and the parton densities are probed. However, it was argued 5:6, that the "natural" scale for jet production in DIS is set by the average $k_{T}^{B}$ of the jets in the Breit frame. Here, $\left(k_{T}^{B}(j)\right)^{2}$ is defined by 
$2 E_{j}^{2}\left(1-\cos \theta_{j P}\right)$, where the subscripts $j$ and $P$ denote the jet and proton, respectively (all quantities are defined in the Breit frame). It can be shown 5 that $\sum_{j} k_{T}^{B}(j)$ smoothly interpolates between the correct limiting scale choices, it approaches $Q$ in the parton model limit and it corresponds to the jet transverse momentum (with respect to the $\gamma^{*}$-proton direction) when the photon virtuality becomes negligible. It therefore appears to be the "natural" scale for multi jet production in DIS and we choose $\mu_{R}^{2}=\mu_{F}^{2}=1 / 4\left(\sum_{j} k_{T}^{B}(j)\right)^{2}$ in Eq. (2) as our standard choice.

Let us first discuss some results for unpolarized dijet cross sections. If not stated otherwise, the lepton and hadron beam energies are 27.5 and $820 \mathrm{GeV}$, respectively. With the previous parameters and GRV parton densities one obtains a LO (NLO) two jet cross section $\sigma^{\text {had }}(2-$ jet$)$ of $1515 \mathrm{pb}(1470 \mathrm{pb})$. Thus the higher order corrections are small. This is essentially due to the relatively large cuts on the transverse momenta of the jets. As mentioned before, the boson-gluon fusion subprocess dominates the cross section and contributes to $80 \%$ to the $\mathrm{LO}$ cross section.

In order to investigate the feasibility of the parton density determination, Fig. 2a shows the Bjorken $x_{B j}$ distribution of the unpolarized two jet exclusive cross section. The gluon initiated subprocess clearly dominates the Compton process for small $x_{B j}$ in the LO predictions. The effective $K$-factor close to unity for the total exclusive dijet cross section is a consequence of compensating effects in the low $x(K>1)$ and high $x(K<1)$ regime.

For the isolation of parton structure functions we are interested in the fractional momentum $x_{a}$ of incoming parton $a(a=q, g)$, however, and in dijet production $x_{B j}$ and $x_{a}$ differ substantially. Denoting as $s_{i j}$ the invariant mass squared of the produced dijet system, and considering two-jet exclusive events only, the two are related by $x_{a}=x_{B j}\left(1+\frac{s_{i j}}{Q^{2}}\right)$. The $s_{i j}$ distribution of Fig. 2b exhibits rather large NLO corrections as well. The invariant mass squared of the two jets is larger at NLO than at LO (the mean value of $s_{i j}$ rising to $620 \mathrm{GeV}^{2}$ at $\mathrm{NLO}$ from $500 \mathrm{GeV}^{2}$ at $\mathrm{LO}$ ).

The NLO corrections to the $x_{B j}$ and $s_{i j}$ distributions have a compensating effect on the $x_{a}$ distribution in Fig. 2c, which shows similar shapes at LO and NLO. At LO a direct determination of the gluon density is possible from this distribution, after subtraction of the calculated Compton subprocess. This simple picture is modified in NLO, however, and the effects of Altarelli-Parisi splitting and low $p_{T}$ partons need to be taken into account more carefully to determine the structure functions at a well defined factorization scale $\mu_{F}$ in NLO.

In the following we discuss some results for polarized dijet production. Our standard set of polarized parton distributions is "gluon, set A" of Gehrmann 
and Stirling $\mathrm{B}$, for which $\int \Delta g=1.8$ at $Q^{2}=4 \mathrm{GeV}^{2}$. Using the same kinematical cuts as before, the LO polarized dijet cross section $\Delta \sigma(2-$ jet $)$ is $-45 \mathrm{pb}$. This negative result for the polarized dijet cross section is entirely due to the boson-gluon fusion process, which is negative for $x_{B j} \lesssim 0.025$. The contribution to the total polarized dijet cross section is $-53 \mathrm{pb}$. The contribution from the quark initiated subprocess is positive over the whole kinematical range and contributes with $8 \mathrm{pb}$ to the resulting dijet cross section. Note, however, that the shape of the $x_{g}$ distribution in the polarized gluon density is hardly (or even not at all) constrained by currently available DIS data (in particular for small $x_{g}$ ). Alternative parametrizations of the polarized gluon distribytions in the small $x_{g}$ region, which are still consistent with all present data 9 , can lead to polarized cross-sections $\Delta \sigma^{\text {had }}$, which are at least a factor two larger than those obtained with "gluon, set A". It is clear from Fig. 2d, that the dijet events are particularly sensitive to this lower $x_{g}$ range. Note, that this fractional momentum distribution is again related to $x_{B j}$ by $x_{a}=x_{B j}\left(1+\frac{s_{i j}}{Q^{2}}\right)$. The corresponding $x_{B j}$ and $s_{i j}$ distributions are not shown here.

\section{Experimental asymmetries}

In order to study the feasibility and the sensitivity of the measurement of the spin asymmetry at HERA, we have assumed polarizations of $70 \%$ for both the electron and the proton beams and statistical errors were calculated for a total luminosity of $200 \mathrm{pb}^{-1}$.

The expected experimental asymmetry $\langle A\rangle=\frac{\Delta \sigma^{\text {had }}(2 \text {-jet })}{\sigma^{\text {had }}(2 \text {-jet })}$ under these conditions is shown in Fig. Ba, as a function of $x_{B j}$ and in Fig. $3 \mathrm{~b}$-d as a function of $x_{g}$. Figures a) and b) correspond to the nominal kinematical cuts defined previously, except for the $Q^{2}$ range which was extended to lower values $2<Q^{2}<2500 \mathrm{GeV}^{2}$. The cross section integrated over all variables is $2140 \mathrm{pb}$, where $82 \%$ of the contribution comes from gluon-initiated events. The asymmetry averaged over all variables is $\langle A\rangle=-0.015 \pm 0.0015$. It is negative at low $x_{B j}$ and becomes positive at $x_{B j}>0.01$.

In figures c) and d), a further cut was made: $Q^{2}<100 \mathrm{GeV}^{2}$. This cut permits to reject the positive contributions to the asymmetry coming from high $Q^{2}$ (equivalent to high $x_{B j}$ ) events, where the contribution of quark-initiated events is higher. All the remaining events were separated in two bins in $s_{i j}$ -the invariant mass of the dijet- and two bins in $y$, as the asymmetry is very sensitive to these two variables. Fig. c) corresponds to low invariant masses $\left(s_{i j}<500 \mathrm{GeV}^{2}\right)$, and Fig. d) to high ones $\left(s_{i j}>500 \mathrm{GeV}^{2}\right)$. Open points show low $y$ values $(y<0.6)$, and closed points, high $y$ values $(y>0.6)$. In 

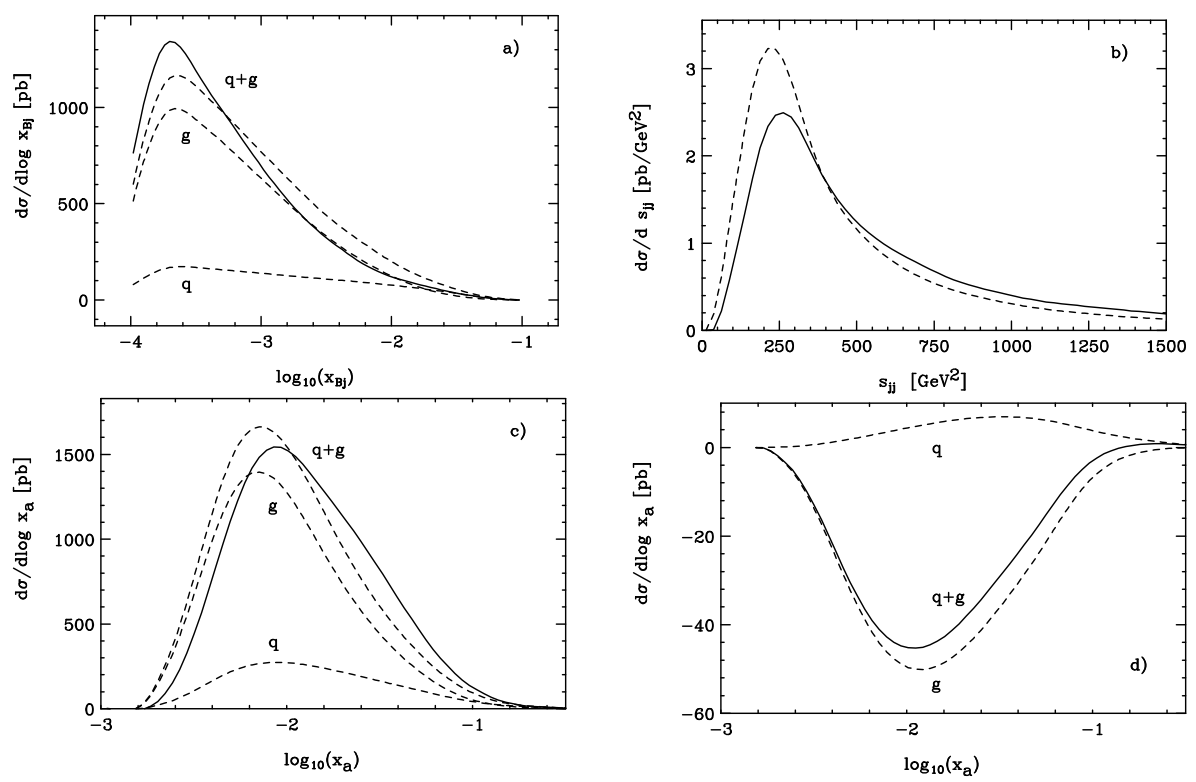

Figure 2: a) Dependence of the unpolarized two-jet cross section on Bjorken $x_{B j}$ for the quark and gluon initiated subprocesses and for the sum. Both LO (dashed) and NLO (solid) results are shown; b) Dijet invariant mass distribution in LO (dashed) and in NLO (solid) for unpolarized dijet production; c) Same as a) for the $x_{a}$ distribution, $x_{a}$ representing the momentum fraction of the incident parton at LO; d) Dependence of the LO polarized two-jet cross section on $x_{a}$ for the quark and gluon initiated subprocesses (dashed) and for the sum (solid).

the best case, the asymmetry reaches values as high as $12 \%$ (Fig. d).

Reducing the beam energy to $410 \mathrm{GeV}$, instead of the nominal $820 \mathrm{GeV}$, does not improve the signal in average, although the mean value of $y$ is higher. The asymmetry signal increases only for a few points around $x_{g}>0.1$, since a higher incident energy probes slightly higher values of $x_{g}$.

The results show that if the assumed luminosity and beam polarizations can be delivered at HERA, the present detectors H1 and ZEUS will be in a comfortable position to measure a spin asymmetry of a few per cent in average, with a few per mil statistical precision. In order to minimize the experimental systematic uncertainties, it is desirable to have in the HERA ring bunch trains of protons with alternate helicity. On the theoretical side, NLO QCD corrections are needed. The NLO corrections reduce the renormalization $\mu_{R}$ and factorization scale $\mu_{F}$ dependence (due to the initial state collinear factor- 

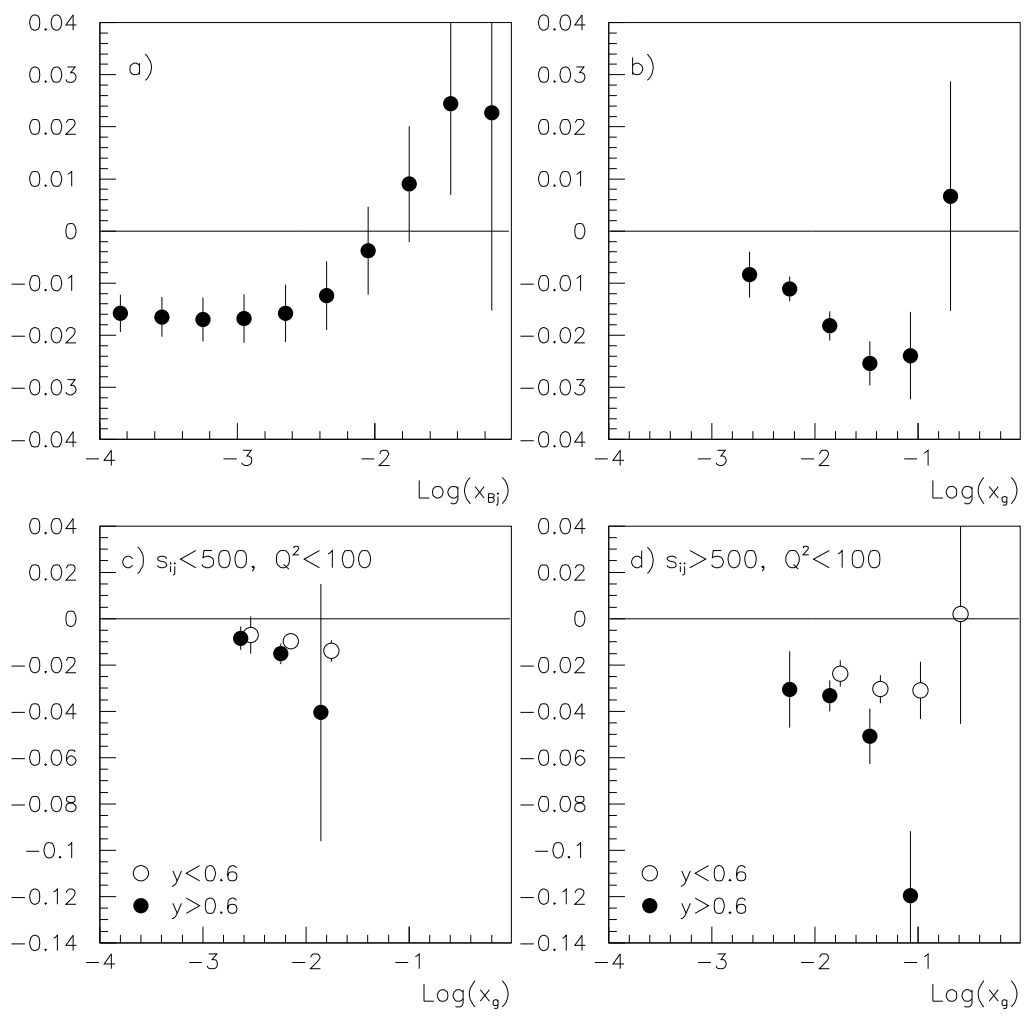

Figure 3: Expected asymmetries as a function of $x_{B j}$ (a) and $x_{g}$ (b-d) for a luminosity of $200 p b^{-1}$ and beam polarizations $P_{e}=P_{p}=70 \%$; Fig.a) and b):nominal cuts; Fig.c) and d): $Q^{2}<100 \mathrm{GeV}^{2}$; In Fig.c) and d) data are separated in bins of $s_{i j}$ and $y$. 
ization) in the LO calculations and thus reliable predictions in terms of a well defined strong coupling constant and scale dependent parton distributions become possible. At the moment, these corrections are only available for unpolarized jet production 36 . One expects for the asymmetry $\left.\langle A\rangle=\frac{\Delta \sigma^{\text {had }}(2-\text { jet }}{\sigma^{\text {had }}(2-\text { jet }}\right)$, that the scale dependence in the individual cross sections partly cancels in the ratio. In fact, varying the renormalization and factorization scales between $\mu_{R}^{2}=\mu_{F}^{2}=1 / 16\left(\sum_{j} k_{T}^{B}(j)\right)^{2}$ and $\mu_{R}^{2}=\mu_{F}^{2}=4\left(\sum_{j} k_{T}^{B}(j)\right)^{2}$ in the LO cross sections introduces an uncertainty for the ratio $A$ of less than $2 \%$, whereas the uncertainty in the individual cross sections is much larger.

In conclusion, the dijets events from polarized electron and polarized proton collisions at HERA can provide a good measurement of the gluon polarization distribution for $0.002<x_{g}<0.2$, the region where $x_{g} \Delta g\left(x_{g}\right)$ is expected to show a maximum.

\section{References}

1. A. Magnon, these proceedings and references therein.

2. H1 coll., Nucl. Phys. B 449, 183 (1995).

3. E. Mirkes and D. Zeppenfeld, hep-ph/9511448, to be published in Phys. Lett. $B$.

4. C. Ziegler and E. Mirkes, Nucl. Phys. B 429, 93 (1994).

5. E. Mirkes and D. Zeppenfeld, hep-ph/9606332, In the proceedings of "QCD and QED in Higher Orders" 1996 Zeuthen Workshop on Elementary Particle Theory, April 22-26, 1996.

6. E. Mirkes and D. Zeppenfeld, these proceedings.

7. M. Glück, E. Reya and A. Vogt, Z. Phys. C 67, 433 (1995).

8. T. Gehrmann and J. Stirling, hep-ph/9512406, to be published in Phys. Rev. D.

9. T. Gehrmann and J. Stirling, private communication. 